\title{
Fresnel Rhombs as Achromatic Phase Shifters for Infrared Nulling Interferometry: First experimental results
}

\author{
Hanot C. ${ }^{a}$, Mawet D. ${ }^{a}$, Loicq J. ${ }^{b}$, Vandormael D. ${ }^{b}$, Plesseria J.Y, , Surdej J. ${ }^{a}$, Habraken S. ${ }^{a, b}$ \\ ${ }^{a}$ Institut d'Astrophysique et de Géophysique, University of Liège, 17 allée du 6 Août, B-4000, \\ Sart Tilman, Belgium; \\ ${ }^{b}$ Centre Spatial de Liège, Avenue du Pré-Aily, B-4031, Liège-Angleur, Belgium
}

\begin{abstract}
One of the most critical units of nulling interferometers is the Achromatic Phase Shifter. The concept we propose here is based on optimized Fresnel rhombs, using the total internal reflection phenomenon, modulated or not. The total internal reflection induces a phase shift between the polarization components of the incident light. We present the principles, the current status of the prototype manufacturing and testing operations, as well as preliminary experiments on a $Z n S e$ Fresnel rhomb in the visible that have led to a first error source assessment study. Thanks to these first experimental results using a simple polarimeter arrangement, we have identified the bulk scattering as being the main error source. Fortunately, we have experimentally verified that the scattering can be mitigated using spatial filters and does not decrease the phase shifting capabilities of the $Z n S e$ Fresnel rhomb.
\end{abstract}

Keywords: Fresnel Rhomb, Achromatic Phase Shifters, nulling interferometry, subwavelength gratings, Zinc Selenide, bulk scattering

\section{INTRODUCTION}

The detection of extrasolar planets and later on the presence of life on them is one of the most interesting and ambitious astrophysical projects for the next decades. Up to now, despite our technological progresses, most exoplanets have been detected using indirect methods. In this context, the next step would be their direct detection and characterization. However, this is a very challenging goal because we have to face two major problems. The first one is the very small angular distance between an exoplanet and its parent star. The second is the large flux ratio between them. For example, an Earth-like exoplanet is typically $\sim 10^{9}$ times fainter than its parent star in the visible spectrum and $\sim 10^{6}$ times in the thermal infrared. One of the solutions that has been proposed by ESA and NASA is the concept of a space-based nulling interferometer. ${ }^{1}$ The principle of nulling interferometry is to combine the coherent beams coming from various telescopes and to introduce a phase shift ( $\pi$ in the simple two-telescope configuration, see Bracewell $1978^{1}$ ) between them in order to produce a destructive interference on the optical axis. Therefore, the light coming from the star is nulled and the signal of the planet can be revealed and analysed.

One of the most critical units of such an interferometer is the phase shifter. Indeed the quality of the destructive interference (the null depth) relies on the optical component ability to induce a very accurate phase shift over the considered wavelength range. The problem is that in order to detect an exoplanet and to perform spectroscopic analysis of its atmosphere, one needs to observe over large spectral bands in the infrared (typically from 6 to 18 microns for the Darwin mission considered by ESA). Therefore, the main challenge consists in conceiving a phase shifter which is able to perform well enough over the whole spectral range. The Achromatic Phase Shifter (APS) concept which is studied in this paper is a vectorial APS proposed by Mawet et al. in $2005^{2,3}$ based on improved Fresnel rhombs. In addition to this very precise application, it is to be emphasized that these improved Fresnel rhombs can also be used as high performance components of a high precision infrared polarimeter.

Further author information: (Send correspondence to Hanot C.)

Hanot C.: E-mail: hanot@astro.ulg.ac.be, Telephone: +32 (0)4 3669768

Mawet D.: E-mail: mawet@astro.ulg.ac.be, Telephone: +32 (0)4 3669757 


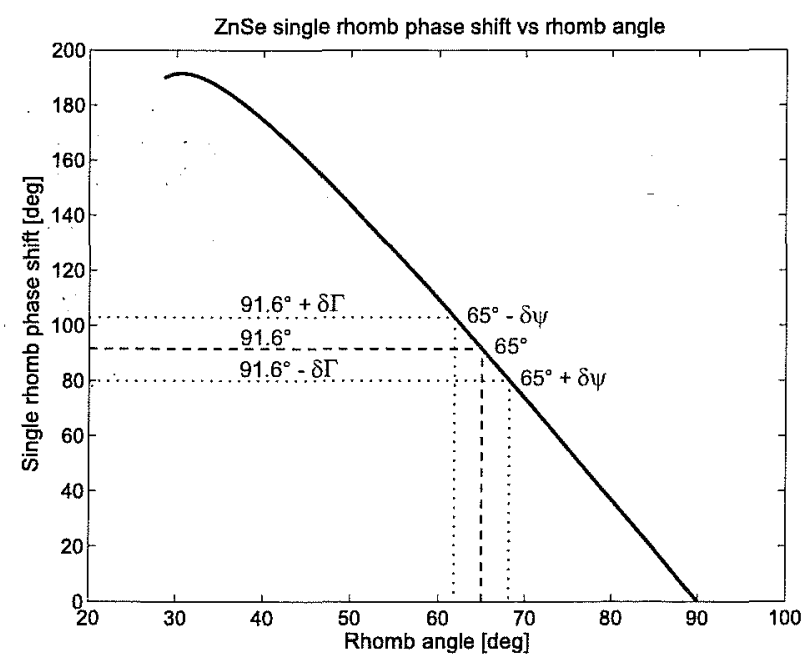

Figure 1. In the incident angle range we use, the retardance depends linearly on $\psi$. Therefore, a double-rhomb geometry allows incidence-angle variations $\delta \psi$ to be compensated by the angle complementarity between the two rhombs and the linearity of the phase shift with respect to the rhomb angle. The linearity is present in the bare Fresnel rhomb or the TIR modulated one.

\section{PRINCIPLES}

The total internal reflection (TIR) phenomenon comes with a phase shift between the vectorial $s$ and $p$ polarization components. This vectorial phase shift takes the following general form ${ }^{4}$

$$
\Delta \Gamma_{s-p}=2 \arctan \left[\frac{\sqrt{\sin ^{2} \psi-n_{t i}^{2}}}{n_{t i}^{2} \cos \psi}\right]-2 \arctan \left[\frac{\sqrt{\sin ^{2} \psi-n_{t i}^{2}}}{\cos \psi}\right]
$$

where $\psi$ is the angle of incidence, greater or equal to $\psi_{c}$, the critical angle defined as $\sin \psi_{c}=n_{t i}=n_{t} / n_{i}$ and where $n_{i}$ and $n_{t}$ are the refractive indices of the incident and emergent media, respectively. This property is exploited in the well-known Fresnel rhombs, which are retarders to be used alone as polarization optics.

\section{MODULATED TOTAL INTERNAL REFLECTION APS}

Classical Fresnel rhombs are limited by the intrinsic index dispersion of the rhomb bulk material. ${ }^{7}$ Engraving a subwavelength grating (often called zero order grating, or ZOG) on the TIR interface or simply depositing a single thin layer of a well chosen material leads to a significant improvement. ${ }^{2,5}$ Indeed, the electromagnetic field evanescent interaction with the optimized micro-structure or thin film allows us to tune the index ratio of Eq. $1, n_{t i}$.

\section{MANUFACTURING}

In this section, we will discuss some manufacturing details for the $Z n S e$ double Fresnel rhomb APS prototype proposed to be one of the selected APS for study, fabrication and test in the framework of the ESA's Darwin R\&D preparatory Nulltimate ${ }^{6}$ activity, which is a project of infrared cryogenic $(100 \mathrm{~K})$ nulling testbed. There are different reasons why we have chosen a rhomb made of $Z n S e$. The first one is that in its polycrystalline state, this material is a priori easy to source and convenient to handle and polish (surface qualities of $\lambda / 30 \mathrm{rms}$ with $\lambda=632.8 \mathrm{~nm}$ are routinely obtained). The second one is that it is compatible with the thickness-adjustable beam splitters of the Nulltimate test bench which are also planned to be in $Z n S e$. Last but not least, a theoretical analysis ${ }^{3}$ has shown that the $Z n S e$ bare Fresnel rhomb with ZOG is meeting the Darwin specification in the 6 $14 \mu \mathrm{m}$ range. 


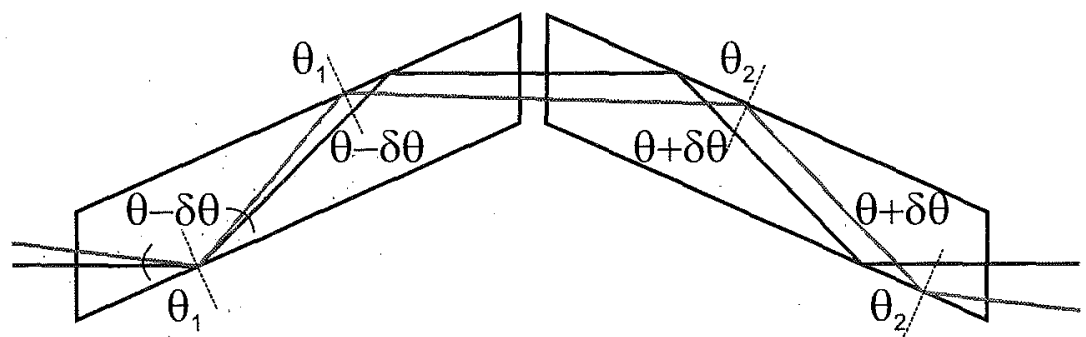

Figure 2. This scheme shows the double-rhomb configuration. $\theta_{1}$ (resp. $\theta_{2}$ ) is the angle of incidence upon the TIR interfaces of the first (resp. second) rhomb.

The drawback of single Fresnel rhombs is that they are known to be very sensitive to incidence angle variations (at the arcmin level). This is a serious drawback in our application since the thermal infrared interferometric beam is likely to slightly diverge because of diffraction while somewhat wandering around because of vibrations. For this reason, we chose the double rhomb configuration for its known insensitivity to incidence angle variations. ${ }^{20}$ Indeed, the pairs of reflections in the two rhombs are complementary (see Fig. 2); an increase in the first two TIR angles due to departure from nominal incidence leads to a decrease in the last two angles. Thus, as the phase shift varies linearly with the TIR angle (see Fig. 1), the retardance changes are cancelled. Therefore, a wider range of incidence angle variations can be tolerated: up to several degrees, ${ }^{2,5}$

As presented in the introduction the contrast between an Earth-like exoplanet and its parent star in the thermal infrared is $10^{-6}$. To detect such kind of planets using nulling interferometry, studies have shown that, thanks to clever modulation schemes, it is sufficient to reach a null depth (ND) of $10^{-5}$ at $7 \mu \mathrm{m} .{ }^{9}$ As our component will be part of a complex instrument, if we want to meet this constraint on the ND, it implies a few $10^{-7}$ for our component. This can be expressed in terms of tolerance on the phase $\operatorname{shift}^{2}\left(N=\frac{\sigma^{2}}{4}\right.$, where $\sigma^{2}$ is the variance of the phase shift over the considered bandpass) which is about $0.1^{\circ}$.

\subsection{ZnSe Rhomb cutting and polishing}

We have conducted cutting and polishing trials of $Z n S e$ rhombs with the appropriate global geometry and dimensions. Results of ZYGO interferometric measurements in terms of surface quality fall comfortably in the specifications, and beyond our expectations with surface figures of $\lambda / 100 \mathrm{rms}(\lambda=632.8 \mathrm{~nm})$. Concerning the roughness, WYKO profilometer measurements gave a rather good $3 \mathrm{~nm} \mathrm{rms}$.

\subsection{TIR Grating manufacturing}

The most appropriate method to manufacture the micro pattern into the rhomb material is based on holographic lithography and dry etching processes. The first one is necessary for masking the parts of the substrates to

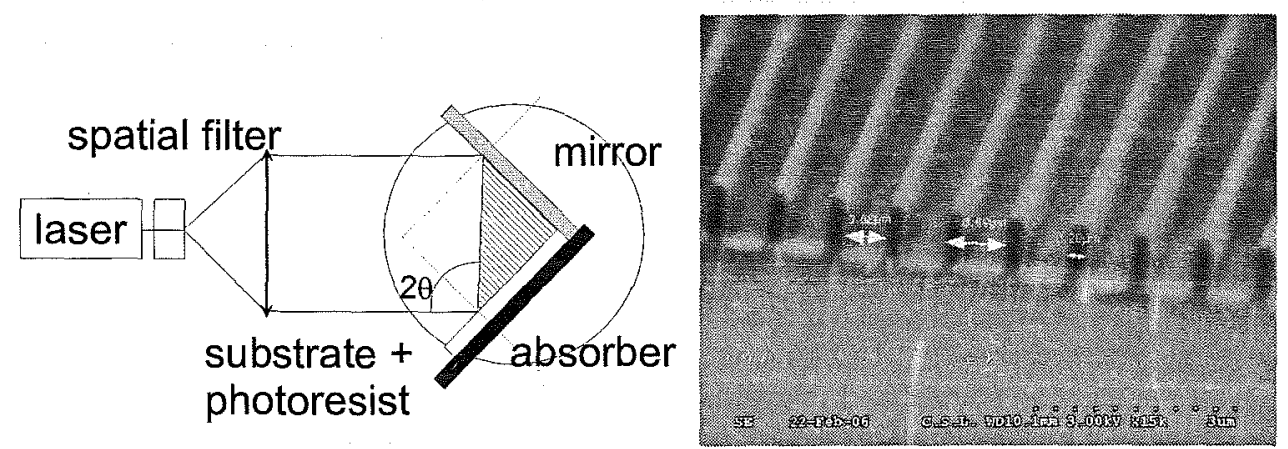

Figure 3. Left: principle of holographic lithography (Lloyd mirror mounting). Right: Micro-pattern on photoresist to be transferred by reactive plasma beam etching into the $Z n S e$ substrate. The period and filling factors correspond to the design specifications, i.e., $\Lambda=0.9 \mu \mathrm{m}$ for the period and $\sim 250 \mathrm{~nm}$ for the feature line. 




Figure 4. Design of the $Z n S e$ double Fresnel rhomb APS mechanical mounts. Left and right images illustrate the practical implementation of the components in the interferometer at $45^{\circ}$ from the test bench table, rotated by $90^{\circ}$ from each other.

be protected during the etching step (Fig. 3, right). Direct laser writing or mask exposure techniques are not applicable onto the rhomb facets since those classical micro-lithography methods are designed for accommodating thin and flat substrates only, such as wafers for micro electronics. The holographic recording (Lloyd mirror mounting, Fig. 3, left) alternative allows us to imprint onto a thick substrate a photoresist mask originated by a fringe pattern created by a two-wave interference. The grating period is inversely proportional to the angle between the two interferometer beams. The typical fringe pattern produced by optical interferences naturally possesses a sine profile. However, a good understanding and control of the recording and developing parameters (as energy dose, development duration, resist thickness, resist optical contrast, etc.) have finally produced reproducible rectangular binary profiles with a controlled filling factor (at the 10-nm level). After its processing, the remaining photoresist pattern serves as a lithographic mask for the subsequent reactive plasma beam etching (RPBE) process, which makes use of both the ballistic effect and chemical reactivity of a beam of reactive ions to transfer structures into a substrate. The various parameters (gas mixtures, beam energy, beam incidence...) characterizing the etching process are optimized for the transfer into various materials. The interest of such a technique comes particularly from its high selectivity, the potential to efficiently etch one material and not another coexisting one, used as a mask. The infrared material ( $Z n S e$ or $C d T e$ ) is engraved using a selective chemistry $\left(\mathrm{CH}_{4} / \mathrm{H}_{2}{ }^{10}\right.$ or chlorine-based ${ }^{11}$ compounds), depending on the crystal phase of the material. Validation trials are currently under progress. They aim at identifying the best process conditions ensuring the most efficient transfer of the diffractive structure from the mask into the material. Highest selectivity, homogeneity and reproducibility are the key factors under optimisation.

\subsection{Mechanical mount}

The two mechanical mounts (one per interferometer arm) of the $Z n S e$ double rhomb must be thoroughly optimized to account for thermo-mechanical effects. Indeed, the component shall be measured at room (298 K) and cryogenic $(100 \mathrm{~K})$ temperatures. It will thus be cooled down and the mount must allow for an efficient heat transfer while compensating differential contractions. The alignment of the two rhombs must be controlled around 3 axis with an arcmin precision. The double rhomb spacing difference between both arms, and thus between the two corresponding mechanical mounts must be below $0.1 \mathrm{~mm}$. The mounts must also account for the $Z n S e$ sensitivity to stress birefringence. In order to quantify the latter to help the mount design, a finite element analysis was conducted and resulted in the conclusion that the rhombs must lay freely at $45^{\circ}$ in their mounts (Fig. 4). Indeed, given the stress-optic coefficient of $Z n S e, C_{\lambda=10.6 \mu m} \approx-12$ brewsters, ${ }^{12}$ even its own weight constraint $\sigma$ can induce a substantial birefringence $\Delta n=C_{\lambda=10.6 \mu \mathrm{m}} \sigma$ which is just within the specifications.

\section{MEASUREMENT OF A $Z N S E$ FRESNEL RHOMB RETARDANCE AT 632.8 NM}

As we have introduced in Sec. 4, a $Z n S e$ Fresnel rhomb under manufacturing at the "Centre Spatial de Liège" (under ESA contract) will be tested on Nulltimate. However, as this infrared test bench is still under development, we have conducted preliminary measurements of the performance of a $Z n S e$ Fresnel rhomb (without subwavelength gratings) at $632.8 \mathrm{~nm}$ with a simple polarimeter arrangement (see Fig. 5). 


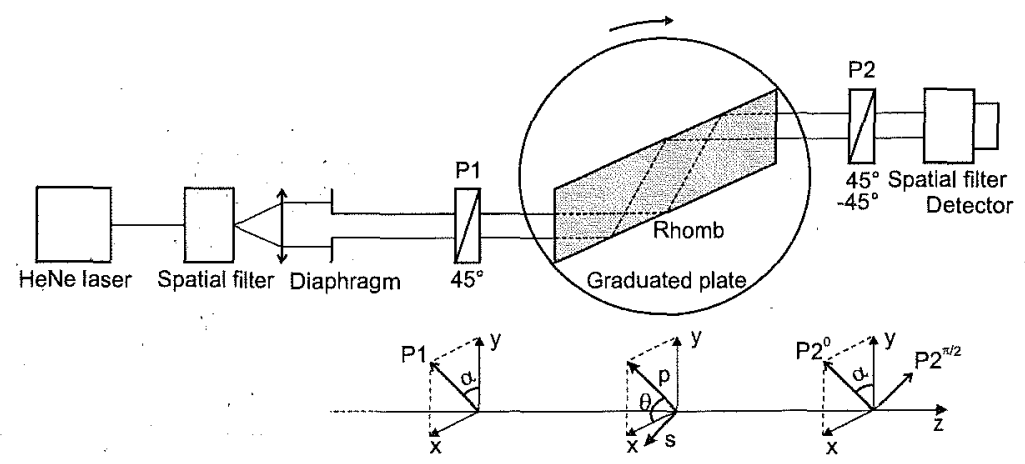

Figure 5. Setup of the optical bench used to perform the $Z n S e$ Fresnel rhomb retardance measurement. Linearly polarized light (through P1) is incident upon the $Z n S e$ rhomb retarder, and the light emerges with an elliptical polarization. The intensities of the two orthogonal polarization states are measured by rotating the output polarizer (P2). $s$ and $p$ are respectively the slow and fast rhomb axis.

\subsection{Principle of the experiment}

Let us compute the theoretical value of the phase-shift as a function of the angle of incidence at TIR surfaces. The phase shift depends on the refractive index $n_{Z n S e}$, the wavelength, the temperature and the angle of incidence $\theta$. In our case, the experiment is done at a constant temperature of about $298 \mathrm{~K}$ and the wavelength is 632.8 $\mathrm{nm}$. Therefore, $n_{Z n S e}=2.59715$ and the phase shift is given by Eq. 1 . The total phase shift at the output of a single ZnSe Fresnel rhomb is twice the phase shift given by Eq. 1 because there are two total internal reflections (TIR) inside the rhomb. Figure 1 illustrates how the retardance varies as a function of the rhomb angle. The phase shift for an angle of $65^{\circ}$ (rhomb angle of our prototype) is about $91.5^{\circ}$.

Once we have this theoretical retardance value, we can measure the experimental one at the output of the rhomb and compare them. The technique used to measure this retardance is based on polarimetry and has been described by C. Yang et al. in $1997 .{ }^{13}$ According to C. Yang ${ }^{13}$ we have

$$
\frac{I^{0}}{I^{\frac{\pi}{2}}}=\frac{\sin ^{2} 2 \alpha \sin ^{4} \theta \sin ^{2}(\Gamma / 2)}{1-4\left(1-\sin ^{2} \alpha \sin ^{2} \theta\right) \sin ^{2} \alpha \sin ^{2} \theta \sin ^{2}(\Gamma / 2)}
$$

where $I^{\frac{\pi}{2}}$ and $I^{0}$ are respectively the intensity of the light at the output of the rhomb when the polarizer and analyzer are rotated by $\frac{\pi}{2}$ (crossed) and $0^{\circ}$ (parallel), where $\alpha$ is the angle between the polarizer and the $y$ axis, and $\theta$ the angle between the rhomb main optical axis $(p)$ and the XZ plane. In our setup (see Fig. 5), $\theta=90^{\circ}$ and we measure the above intensity ratio for different values of $\alpha$ around $45^{\circ}$ where we have an equal distribution in both polarizations.

Equation 2 gives us a direct relation between the phase shift and the flux ratio $\frac{I^{0}}{I^{\frac{\pi}{2}}}$. To get the induced retardance with a good accuracy, we performed several measurements for different orientations of the polarizer and fitted the theoretical curve given by Eq. 2 (where the phase shift $\Gamma$ is the only unknown) on them.

\subsection{Error budget}

Unfortunately, in practice, we always have to deal with parasitic effects. We have therefore to discuss the different sources of errors of this experiment and constrain their influences on the measured phase shift. The different error sources that we have identified are:

- absorption,

- induced birefringence,

- positioning error on the incident angle,

- positioning error on both polarizer and analyzer $(\Delta \alpha)$, 
- positioning error on $\theta$,

- scattering,

- presence of ghosts due to spurious reflections at the $Z n S e /$ air interfaces.

\subsubsection{Absorption}

$Z n S e$ is highly transparent in the infrared window but starts to absorb in the visible $(\alpha=0.037247$ at 632.8 $\mathrm{nm}) .{ }^{14}$ Therefore, it is possible that our measurements are slightly perturbed by absorption $\left(\frac{I}{I_{0}}=e^{-\alpha Z} \simeq 99.5 \%\right.$ for $Z=0.1 \mathrm{~m}$ ).

What we have to carefully study is the influence of the complex refractive index on the retardance using Eq. 1. The imaginary part of the refractive index $n_{i}$ being 0.5627 , the impact on the retardance is about $0.4^{\circ}\left(91.94^{\circ}\right.$ instead of $91.54^{\circ}$ ).

\subsubsection{Induced birefringence}

We need as little as possible birefringence in our rhomb since the beam travels a long way inside it. Though $Z n S e$ has no inherent birefringence properties, it is subjected to stress-induced birefringence (proportionally to the stress-optic coefficient). According to Goldstein et al., ${ }^{15}$ the induced birefringence for $Z n S e$ can be expressed by

$$
\begin{aligned}
& \Delta n_{1}(y)=-\frac{1}{2}\left(n^{0}\right)^{3}\left[\sigma_{1}(y) \pi_{11}+\sigma_{2}(y) \pi_{12}\right] \\
& \Delta n_{2}(y)=-\frac{1}{2}\left(n^{0}\right)^{3}\left[\sigma_{1}(y) \pi_{12}+\sigma_{2}(y) \pi_{11}\right]
\end{aligned}
$$

where $\Delta n_{i}(y)$ is the variation of refractive index as a function of height along the sample $(\mathrm{y})$ in the $\mathrm{i}^{\mathrm{th}}$ polarization direction, $n^{0}$ the refractive index of $Z n S e$ at $632.8 \mathrm{~nm}$ and $\pi_{m n}$ are the stress-optic coefficients.

The experimental values ${ }^{15}$ for the stress-optic coefficients $\pi_{11}$ and $\pi_{12}$ are $-1.4810^{-12} \mathrm{~m}^{2} / \mathrm{N}$ and $0.2210^{-12}$ $\mathrm{m}^{2} / \mathrm{N}$. If now, we assume that in the configuration of our experiment, only the main stress component $\sigma_{1}$ is significant, we have

$$
\begin{aligned}
& \Delta n_{1}(y)=\frac{1}{2}(2.58)^{3} 1.4810^{-12} \sigma=12.710^{-12} \sigma \\
& \Delta n_{2}(y)=-\frac{1}{2}(2.58)^{3} 0.2210^{-12} \sigma=-1.910^{-12} \sigma
\end{aligned}
$$

If we consider a rhomb lying on a face under its proper weight $(\sim 0.150 \mathrm{Kg})$, we find that the difference in the phase shift between the two polarizations is $1^{\circ}$ at the basis of the rhomb and $0.5^{\circ}$ at mid height of the rhomb (where the beam passes). Note that the influence of induced birefringence on the phase shift is proportional to the stress-optic coefficients but inversely proportional to the wavelength. Therefore, as the stress-optic coefficients do not change so much from the visible $(632.8 \mathrm{~nm})$ to the Darwin wavelength range $([6-18] \mu \mathrm{m})$, the birefringence error is divided by ten at the wavelengths the rhomb has been designed for.
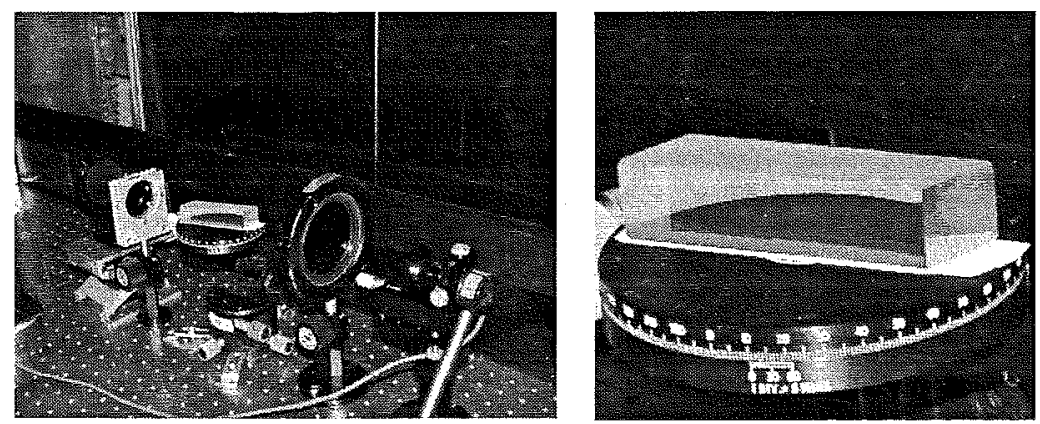

Figure 6. Left: Picture showing the setup of the bench. Right: Picture of the $Z n S e$ rhomb prototype 

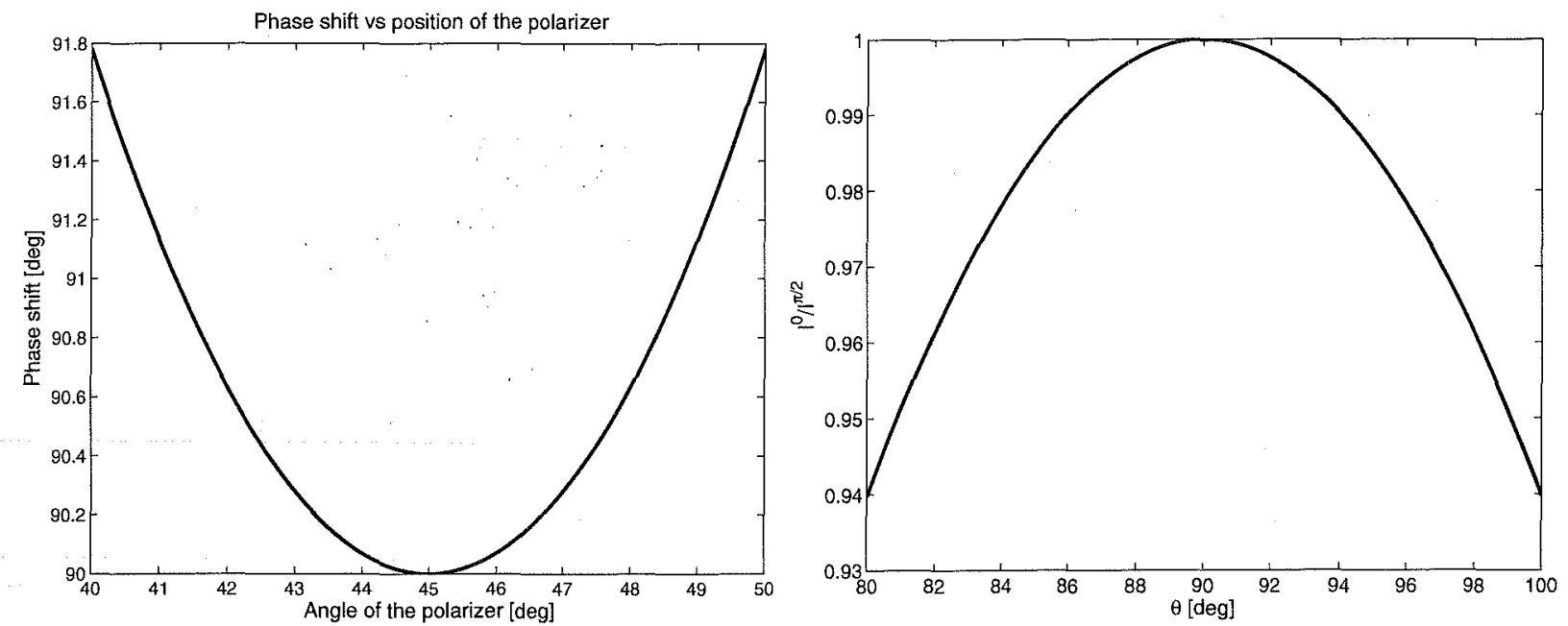

Figure 7. Left: plot of the phase shift as a function of the angle of polarization $\alpha$. Right: intensity ratio as a function of $\theta$

\subsubsection{Precision on the incident angle}

To compute the influence of the incident angle on the retardance, we can simply use Eq. 1. Therefore, if we look at Fig. 1 which illustrates this equation, we can see that, for incident angles around $65^{\circ}$, the phase shift, linearly depends on the incident angle $(\delta \Gamma=\gamma \delta \psi$ where $\gamma$ is the slope of the curve). Unfortunately, as our experiment is a first assessment, we only have a precision on this angle of 5'. The impact on the retardance is therefore about $0.3^{\circ}$. As explained in Sec. 4, the precisions we would like to reach in order to validate the APS for the specifications of Darwin is $0.1^{\circ}$. This will require a precision on the incident angle of $1^{\prime} .7$. We are aware of this critical point and we are currently developing a new bench that will satisfy this requirement.

\subsubsection{Positioning of the polarizer and analyzer axis}

Because the polarizers we use cannot reach a very good positioning accuracy $\left(\Delta \alpha \simeq 0.1^{\circ}\right)$, we have to carefully study the influence of the precision of both the polarizer and analyzer on the phase shift measurement. Thus we want to study the variations of $\Gamma$ around the position $\theta=\frac{\pi}{2}$ and $\alpha=\frac{\pi}{4}$. For $\theta=\frac{\pi}{2}$, equation (2) becomes

$$
\Gamma=2 \arcsin \sqrt{\frac{1}{4\left(1-\sin ^{2} \alpha\right) \sin ^{2} \alpha+\frac{I^{0}}{I^{\frac{\pi}{2}}} \sin ^{2} 2 \alpha}}
$$

The problem with this equation is that the ratio $\frac{I^{0}}{I^{\frac{\pi}{2}}}$ is unknown. Therefore, we make the assumption that $\Gamma \simeq \frac{\pi}{2}$ and $\alpha \simeq \frac{\pi}{4}$. Therefore, $\frac{I^{\circ}}{I^{\frac{\pi}{2}}}=\tan ^{2}(\Gamma / 2)=1$. Using this simplification, Eq. 7 becomes

$$
\Gamma=2 \arcsin \sqrt{\frac{1}{4\left(1-\sin ^{2} \alpha\right) \sin ^{2} \alpha+\sin ^{2} 2 \alpha}}
$$

Figure 7 (left) represents the phase shift $\Gamma$ as a function of $\alpha$. For polarizer positioning precision of $1^{\circ}$ (around $45^{\circ}$ ), the variation on the phase shift is about $0.1^{\circ}$ and vary as the square of the positioning accuracy. Therefore, we conclude that this error source is not so critical, even at the precision level of our polarizers.

\subsubsection{Influence of $\theta$}

The fifth error source that we consider is the influence of $\theta$ on our measurements. If we assume that $\alpha=45^{\circ}$ and that $\Gamma=90^{\circ}$ Eq. 2 can be re-written as follows 


$$
\frac{I^{0}}{I^{\frac{\pi}{2}}}=\frac{\frac{1}{2} \sin ^{4} \theta}{1-\left(1-\frac{1}{2} \sin ^{2} \theta\right) \sin ^{2} \theta}
$$

This dependency on $\theta$ is plotted in figure 7 (right). We see that the variation of the ratio due to a misalignment of the rhomb axis $(\theta)$ is below the accuracy that is possible to reach with our present measurements $(\sim 10 \%$ on $\left.\frac{I^{0}}{I^{\frac{\pi}{2}}}\right)$. Therefore, we conclude that the position of the rhomb along $\theta$ is not really relevant for the measurement of the retardance. However, if we want to reach a very high rejection ratio, the positioning of the rhomb along this axis becomes important. Indeed one can compute its influence on the ND as follows: $N D=(1+\sin \Delta \theta)(1-$ $\cos \Delta \theta) / 2$. Therefore, to fulfill the constraint on $N D$ for Darwin, we must impose that $\Delta \theta \leq 2$ arcmin.

\subsubsection{Scattering}

Two kinds of scattering are present in this experiment: surface and bulk scattering. The bulk scattering is also called extrinsic absorption (by opposition to intrinsic absorbtion which is the classical absorbtion of a wave by a material). Extrinsic absorbtion is the scattering due to impurities, structural defects, and deviation from stoichiometry. It strongly depends on the material preparation procedure. ${ }^{16}$ This phenomenon is therefore affected by a very high variability. We will, in this section, try to assess scattering thanks to the combination of measurements and a simple model. In order to measure the amount of scattered light, we first have to assume that the scattered light is depolarized ${ }^{17}$ and that the bulk scattering is linearly dependent on the thickness. However, according to S. Schröder et al. $2006^{18}$ this assumption is only strictly correct up to a thickness of 10 $\mathrm{mm}$. After measuring the nominal extinction ratio $\left(\mathrm{ER}=\frac{\mathrm{I}^{\text {crossed }}}{\text { Iparallel }}\right)$ with our polarizers to be equal to $10^{-5}$, we can compare it to the extinction ratio deterioration obtained by introducing $Z n S e$ samples of different thicknesses between the polarizers. The two samples we use have been manufactured from the same block of $Z n S e$ and have the same polishing properties (the roughness of the surfaces is $3 \mathrm{~nm}$ RMS) as the rhombs. The first sample has a thickness of $20 \mathrm{~mm}$, and we place its interfaces perpendicular to the beam (see Fig. 8, left). Therefore, we observe surface scattering on two surfaces (roughness: $3 \mathrm{~nm}$ RMS) and bulk scattering over $20 \mathrm{~mm}$. The second sample has a thickness of $3 \mathrm{~mm}$, thus we also have surface scattering on two surfaces (roughness: $3 \mathrm{~nm}$ RMS), but we only have bulk scattering through $3 \mathrm{~mm}$. Therefore, the difference between the two samples is only due to $17 \mathrm{~mm}$ bulk scattering.

In practice, we only measure a fraction $\beta$ of the total bulk-scattered light and $\gamma$ of the surface-scattered light. The measured ER are summarized in the following table.

\begin{tabular}{lc}
\hline Configuration & Extinction ratio (ER) \\
\hline Polarizers alone & $10^{-5}$ \\
Sample of $3 \mathrm{~mm}$ & $2.510^{-3}$ \\
Sample of $20 \mathrm{~mm}$ & $10^{-2}$ \\
\hline
\end{tabular}

Using this table, the situation can be modelled by the following simple equations

$$
\begin{array}{r}
2 \gamma S S+3 \beta B S=0.0025 \\
2 \gamma S S+20 \beta B S=0.01
\end{array}
$$

where $B S$ is the bulk scattering per $\mathrm{mm}$. The value of $S S$ is determined using the assumption of the Total Integrated Scattering formula (TIS): $S S=0.355 \%$ / surface. There only remain two unknowns in this system: $\beta$ and $B S$ so we have to find a way to characterize bulk-scattering in $Z n S e$. If we consider that bulk scattering is induced by specular reflection at intergrain boundaries ${ }^{19}$ and that these interfaces are randomly oriented, we can evaluate $\beta$ by computing the reflectance as a function of the angle of incidence $\theta$. This reflectance is obtained by multiplying the Fresnel relation of both polarizations by a weighting $\operatorname{coefficient} \cos \theta$ (where $\theta$ is the local incident angle at the grain interface) meaning that an interface $\Delta S$ has an effective cross-section $\Delta S \cos (\theta)$ for the beam (see Figure 8). As we can see in Figure 9, the scattered beam is not so much diffused. Therefore, as 
our detector has a field of view of about $2^{\circ}\left(1^{\circ}\right.$ away from the optical axis) we measure about $2 / 3$ of the total bulk-scattered flux. If we use this value for $\beta$ in equation 11, we get a total value for the bulk scattering of $6 \%$ (for an usual use of the rhomb at $632.8 \mathrm{~nm}$ ).

$Z n S e$ exists in two different states: monocrystalline and polycrystalline. Our prototype has been manufactured using polycrystalline $Z n S e$. Studies carried out at wavelengths of $1.1 \mu \mathrm{m}$ and $10 \mu \mathrm{m}$ have shown that the grain boundary is an interlayer with a perturbed structure containing intergrain dislocations and impurities. ${ }^{19}$ According to A. N. Bryzgalov et al. $2003,{ }^{19}$ the basic mechanism of scattering inside polycrystalline $\mathrm{ZnSe}$ can be modelled by a set of chaotic grain orientations characterized by a variation of refractive index at the grain boundaries $\left(\Delta n \simeq 10^{-3}\right.$ at $\left.10 \mu \mathrm{m}\right)$. Using this, the light transmission at a given wavelength is defined by the coefficient $\mu$ of radiation-flux attenuation: $\mu=d^{-1} \bar{R}$, where $\bar{R}$ is the reflectance averaged over the angles of incidence and $d$ is the average grain size (50 $\mu \mathrm{m}$ for the sample we have). Therefore, the bulk scattering (see Fig. 8) can be defined as

$$
B S=1-e^{-\mu L}
$$

If now, we try to fit the above bulk scattering model on our experimental results letting $\Delta n$ as a free parameter, we find that the $6 \%$ of bulk scattering correspond to $\Delta n=5 \cdot 10^{-4}$ which is consistent with the value given by N. Bryzgalov et al. ${ }^{19}$ (see Fig. 9, right). Indeed, $\Delta n$ can vary from one piece of $Z n S e$ to an other due to the manufacturing process. As we can see on Fig. $9, \Delta n=10^{-3}$ would imply $12 \%$ of bulk scattering instead of $6 \%$. Note that the total amount of scattering has to be multiplied by two for the double rhomb configuration.

We can therefore reasonably think that a model based on the TIS formula and on bulk scattering induced by specular reflection at the grain boundaries is consistent. For a use of the rhomb in the visible we have thus: $\beta=66 \%, B S=6 \%$ and $S S=0.355 \%$ / surface. Fortunately, this error source can be mitigated using spatial filter (which will be the case on Nulltimate) and does not decrease the retardance capabilities of the $Z n S e$ Fresnel rhomb. Note that the use of monocrystalline $Z n S e$ should also largely improve the bulk-scattering.

To conclude this scattering assessment study, let us extrapolate from the error values obtained in the visible, the importance of the scattering in the Darwin wavelength range. This value strongly depends on the size of the grains. If the size of the grains is well above the wavelength, the bulk scattering does not vary with the wavelength. But if the size of the grains is similar to the wavelength, the bulk scattering proportionally varies to $\lambda^{-1}$ (Mie scattering). Concerning the surface scattering, it is proportionnal to $\lambda^{-2}$. Therefore, in the [6-18 $\left.\mu \mathrm{m}\right]$ Darwin wavelength range, the bulk scattering should be about the same (1-10\%) but surface scattering error is divided by 100 and becomes negligible. However, only $10 \mu \mathrm{m}$ measurements would allow us to quantify exactly

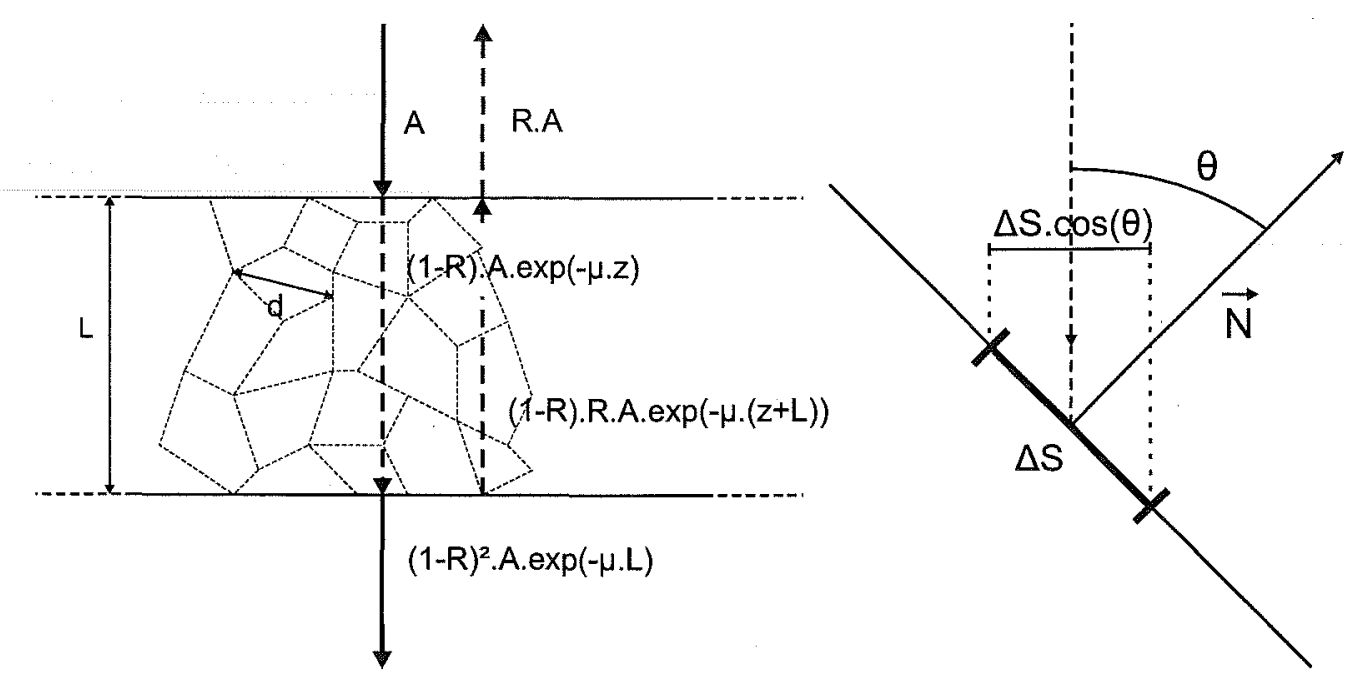

Figure 8. Left: Scheme showing the geometry optics model that we have used. In our case, $d=50 \mu \mathrm{m}$ and $L=10 \mathrm{~cm}$. Right: Effective cross-section of the grains seen by the beam as a function of $\theta$ 

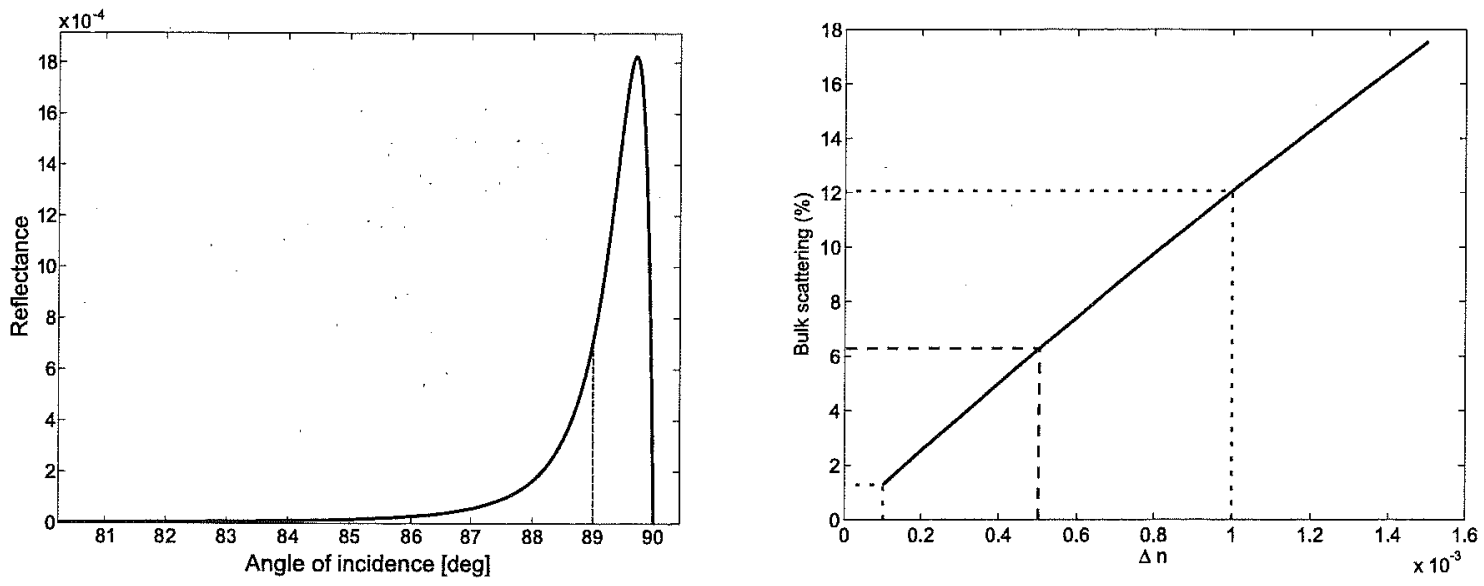

Figure 9. Left: Reflectance as a function of $\theta$. Right: Percentage of bulk scattering inside the rhomb as a function of $\Delta n$ (the variation of refractive index at the intergrain boundaries).

the scattering in the thermal infrared. We are currently developing in Liège a new generation of polarimetric bench that will allow us to perform this scattering study.

\subsubsection{Ghosts}

Another very critical point is the presence of ghosts induced by spurious reflections inside the rhomb. The problem comes from the fact that we use a monochromatic coherent source for the experiment. Therefore, as the length of the path at the output of the rhomb is different for the main beam and for the ghosts, the different beams interfere together and strongly impact the retardance. Indeed, according to Rochford et al. $(1997),{ }^{20}$ for a perfectly coherent source, the change in retardance resulting from spurious reflections $\Delta \delta(R)$ can be found by summing up the individual fields that contribute to the transmitted fields. For a reflectance $R$, we have

$$
\Delta \delta[R, \phi(t)]=\tan ^{-1}\left\{\frac{R \sin \left[\phi(t)-\delta_{0}\right]}{1-R \cos \left[\phi(t)-\delta_{0}\right]}\right\}-\tan ^{-1}\left\{\frac{R \sin \left[\phi(t)-\delta_{0}\right]}{1-R \cos \left[\phi(t)-\delta_{0}\right]}\right\}
$$

where $\delta_{0}$ is the device TIR retardance, and $\phi(t)$ is the round-trip phase which typically varies with time and temperature. If we assume a uniform distribution of $\phi(t)$ in the $[0,2 \pi]$ interval, we find the probability density function for $\Delta \delta(R): P(\Delta \delta) .{ }^{20}$ The mean error in this case is zero. In the case of $\delta_{0}=90^{\circ}$ and of small reflectance, the standard deviation is about $\sqrt{2} R$. During our experiment, we have measured the influence of the ghosts on the retardance to be above $10^{\circ}$.

\subsubsection{Conclusions on the error budget}

Let us summarize the impact of the different error sources in the following table.

\begin{tabular}{lc}
\hline Error source & Impact on the retardance \\
\hline Absorption & $\sim 0.4^{\circ}$ \\
Birefringence & $\sim 0.5^{\circ}$ \\
Incident angle & $\sim 0.3^{\circ}$ \\
Position of the polarizer and analyzer & $\sim 0.1^{\circ}$ \\
Precision on $\theta$ & $\sim 0.1^{\circ}$ \\
Scattering & $/$ \\
Ghosts & $>10^{\circ}$ \\
\hline Total & $\sim 1.4^{\circ}$ \\
\hline
\end{tabular}

In this table, we have not taken into account the influence of scattering and ghost in the error budget because both can be suppressed using respectively a spatial filter and a tilt of the rhomb. 


\subsection{Results}

In order to keep a beam as free from optical defects as possible and to avoid the influence of ghosts in our measurements, we have used a bench with two (input/output) spatial filters and tilted the rhomb by $1^{\circ}$ from the optical axis.

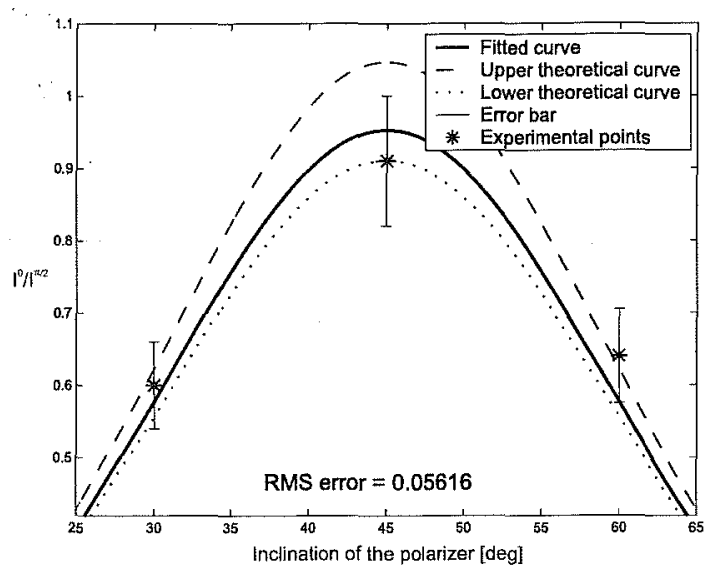

Figure 10. $Z n S e$ single rhomb retardance measurement. Dashed end dotted lines: upper and lower limits of the expected retardance vs polarizer inclinations. Dashed line: fitted curve corresponding to a retardance of $88.8^{\circ} \pm 1.5^{\circ}$. The experimental points are represented by asterix. The RMS error of the fit is 0.05616 and corresponds to a phase error of $1.5^{\circ}$, which is fully compliant with our setup precision.

The rhomb TIR angle of $65^{\circ} \pm 0.1^{\circ}$ theoretically should provide a $91^{\circ} \pm 0.5^{\circ}$ phase shift at $632.8 \mathrm{~nm}$. However, tilting the rhomb of about $1^{\circ}$ was necessary to get rid of the annoying ghost, in agreement with a ray-tracing analysis that concluded to the necessity of wedging the final rhombs. ${ }^{3}$ We have then measured a phase shift value of $88.8^{\circ} \pm 1.5^{\circ}$ (see Fig. 10) (uncertainty of the optical setup) which is consistent with the tilt-corrected theoretical value of $90.2^{\circ} \pm 0.5^{\circ}$ (uncertainty of the measured rhomb angle). Given the respective uncertainties in our experiment (systematics), we can extrapolate an upper boundary for the null depth, estimated at a few $10^{-4}$, which is very encouraging.

\section{CONCLUSION}

To summarize, we have presented some manufacturing key points as well as first preliminary measurements for the $Z n S e$ Fresnel rhomb prototype. This first error budget study performed in the visible shows very promising results. Indeed, with a simple polarimeter setup, we have experimentally proven that this APS concept was able to reach a null depth better than $10^{-4}$, a value limited only by the accuracy of our bench. The two major error sources have been identified to be scattering and ghosts. However these errors can be mitigated respectively using spatial filters and by tilting or wedging the rhomb. It is important to be very careful about the interpretation of the bulk scattering analysis. Indeed, this is a very complex topic which can really vary from one sample of $Z n S e$ to another. What we have done here is a first experimental study of the performances of our Fresnel rhomb. It cannot be interpreted as an average behavior of a standard sample of $Z n S e$. A new generation of polarimetric bench is currently under development at the "Institut d'Astrophysique et de Géophysique de l'Université de Liège" (IAGL) in Belgium. This bench will provide us a much better accuracy and will allow us to perform a deeper analysis of the $Z n S e$ Fresnel rhomb before its final test in the thermal infrared on Nulltimate.

\section{ACKNOWLEDGMENTS}

The authors acknowledge the financial support of ESA and of the Euro Space Society. 


\section{REFERENCES}

1. R. N. Bracewell, "Detecting nonsolar planets by spinning infrared interferometer," Nature 274, pp. 780-781, Aug. 1978.

2. D. Mawet, C. Lenaerts, P. Riaud, J. Surdej, S. Habraken, and D. Vandormael, "Use of subwavelength gratings in TIR incidence as achromatic phase shifters," Opt. Express 13, pp. 8686-8692, 2005.

3. D. Mawet, C. Hanot, C. Lenaerts, P. Riaud, D. Vandormael, J. Loicq, K. Fleury, J. Y. Plesseria, D. Defrère, J. Surdej, and S. Habraken, "Fresnel Rhombs as Achromatic Phase Shifters for Infrared Nulling Interferometry," Submitted to Opt. Express.

4. M. Born and E. Wolf, Reflection and refraction of a plane wave, Principles of Optics, seventh edition ed., 1997.

5. D. Mawet, C. Lenaerts, P. Riaud, D. Vandormael, J. Loicq, D. Verstraeten, K. Fleury, S. Habraken, and J. Surdej, "Infrared achromatic phase shifters using modulated total internal reflection," Proc. SPIE 6268, pp. 6268-6279, 2006.

6. A. Labèque, B. Chazelas, F. Brachet, C. Commeaux, P. Blache, , A. Léger, M. Ollivier, T. Lepine, and C. Valette, "Nulltimate project: building and testing, at low temperature, achromatic phase shifters to prepare the Darwin mission," Proc. SPIE 5491, pp. 999-1010, 2004.

7. R. Anderson, "Quarterwaveplate and Fresnel rhomb compared in the 10-Mum CO2 laser emission region," Appl. Opt. 27, pp. 2746-2747, 1988.

8. K. Rochford, A. Rose, P. Williams, C. Wang, I. Clarke, P. Hale, and G. Day, "Design and performance of a stable linear retarder," Appl. Opt. 36, pp. 6458-6465, 1997.

9. B. Chazelas, F. Brachet, P. Bord, B. Mennesson, M. Ollivier, O. Absil, A. Labque, C. Valette, and A. Léger, "Instrumental stability requirements for exoplanet detection with a nulling interferometer: variability noise as a central issue," Appl. Opt. 45, pp. 984-992, 2006.

10. W. R. Chen, S. J. Chang, Y. K. Su, W. H. Lan, A. C. H. Lin, and H. Chang, "Reactive Ion Etching of ZnSe, ZnSSe, ZnCdSe and ZnMgSSe by H2/Ar and CH4/H2/Ar," Jpn. J. Appl. Phys. 39, pp. 3308-3313, 2000 .

11. K. Kurisu, T. Hirai, K. Fuse, T. Okada, K. Ebata, A. Ikegaya, and M. Tatsumi, "Development of a Diffractive Optical Element for Laser Processing," SEI Technical Review 53, pp. 86-91, 2002.

12. G. R. Mariner and K. Vedam, "Stress-optic coefficient of ZnSe at 10.6 microns," Appl. Opt. 20, pp. 28782879, Sept. 1981.

13. C. Yang and P. Yeh, "Artificial uniaxial and biaxial dielectrics with the use of photoinduced gratings," Journal of Applied Physics 81, pp. 23-29, Jan. 1997.

14. D. C. Harris, "Durable 3 - $5 \mu \mathrm{m}$ transmitting window materials," Infrared Physics 8 technology, 2006.

15. L. Goldstein, J. Thompson, J. Schroeder, and J. Slattery, "Stress-optic coefficients of ZnSe," Appl. Opt. 14, pp. 2432-2434, 1975.

16. E. M. Gavrushchuk, "Polycrystalline Zinc Selenide for IR Optical Applications," Inorganic Materials 39, pp. $883-898,2003$.

17. J. M. Bueno, E. Berrio, M. Ozolinsh, and P. Artal, "Degree of polarization as an objective method of estimating scattering," J. Opt. Soc. Am. A 21, pp. 1316-1321, 2004.

18. S. Schröder, M. Kamprath, A. Duparré, A. Tünnermann, B. Kühn, and U. Klett; "Bulk scattering properties of synthetic fused silica at $193 \mathrm{~nm}, "$ Opt. Express 14, pp. 10537-10549, 2006.

19. A. N. Bryzgalov, V. V. Musatov, and V. V. Buz'ko, "Optical Properties of Polycrystalline Zinc Selenide," Semiconductors 38, pp. 310-312, 2003.

20. K. Rochford, A. Rose, P. Williams, C. Wang, I. Clarke, P. Hale, and G. Day, "Design and performance of a stable linear retarder," Appl. Opt. 36, pp. 6458-6465, 1997. 